\title{
Methodology of Calculation of Dynamics and Hydromechanical Characteristics of Heavy-Loaded Tribounits, Lubricated with Structurally-Non-Uniform and Non-Newtonian Fluids
}

\author{
Juri Rozhdestvenskiy, Elena Zadorozhnaya, Konstantin Gavrilov, \\ Igor Levanov, Igor Mukhortov and Nadezhda Khozenyuk \\ South Ural State University \\ Russia
}

\section{Introduction}

Friction units, in which the sliding surfaces are separated by a film of liquid lubricant, generally, consist of three elements: a journal, a lubricating film and a bearing. Such tribounits are often referred to as journal bearings. Tribounits with the hydrodynamic lubrication regime and the time-varying magnitude and direction of load character are hydrodynamic, heavy-loaded (unsteady loaded). Such tribounits include connecting-rod and main bearings of crankshafts, a "piston-cylinder" coupling of internal combustion engines (ICE); sliding supports of shafts of reciprocating compressors and pumps, bearings of rotors of turbo machines and generators; support rolls of rolling mills, etc. The presence of lubricant in the friction units must provide predominantly liquid friction, in which the losses are small enough, and the wear is minimal.

The behavior of the lubricant film, which is concluded between the friction surfaces, is described by the system of equations of the hydrodynamic theory of lubrication, a heat transfer and friction surfaces are the boundaries of the lubricant film, which really have elastoplastic properties. During the simulation and calculation of heavy-loaded bearings researchers tend to take into account as many geometric, force and regime parameters as possible and they provide adequacy of the working capacity forecast of the hydrodynamic tribounits on the early stages of the design.

\section{The system of equations}

In the classical hydrodynamic lubrication theory of fluid the motion in a thin lubricating film of friction units is described by three fundamental laws: conservation of a momentum, mass and energy. The equations of motion of movable elements of tribounits are added to the equations which are made on the basis of conservation laws for heavy-loaded bearings. 
The problem of theory of hydrodynamic tribounits is characterized by the totality of methods for solving the three interrelated tasks:

1. The hydrodynamic pressures in a thin lubricating film, which separates the friction surfaces of a journal and a bearing with an arbitrary law of their relative motion, are calculated.

2. The parameters of nonlinear oscillations of a journal on a lubricating film are detected and the trajectories of the journal center are calculated.

3. The temperature of the lubricating film is calculated.

The field of hydrodynamic pressures in a thin lubricating film depends on:

- the relative motion of the friction surfaces;

- the temperature parameters of the tribounit lubricant film during the period of loading, sources of lubricant on these surfaces are taken into account;

- the elastic deformation of friction surfaces under the influence of hydrodynamic pressure in the lubricating film and the external forces;

- the parameters of the nonlinear oscillation of a journal on the lubricating film with a nonstationary law of variation of influencing powers;

- the supplies-drop performance of a lubrication system;

- the characteristics of a lubricant, including its rheological properties.

Complex solution of these problems is an important step in increasing the reliability of tribounits, development of friction units, which satisfy the modern requirements. However, this solution presents great difficulties, since it requires the development of accurate and highly efficient numerical methods and algorithms.

The simulation result of heavy-loaded tribounits is accepted to assess by the hydromechanical characteristics. These are extreme and average per cycle of loading values for the minimum lubricant film thickness and maximum hydrodynamic pressure, the meanflow rate through the ends of the bearing, the power losses due to friction in the conjugation, the temperature of the lubricating film. The criterions for a performance of tribounits are the smallest allowable film thickness and maximum allowable hydrodynamic pressure.

\subsection{Determination of pressure in a thin lubricating film}

The following assumptions are usually used to describe the flow of viscous fluid between bearing surfaces: bulk forces are excluded from the consideration; the density of the lubricant is taken constant, it is independent of the coordinates of the film, temperature and pressure; film thickness is smaller than its length; the pressure is constant across a film thickness; the speed of boundary lubrication films, which are adjacent to friction surfaces, is taken equal to the speed of these surfaces; a lubricant is considered as a Newtonian fluid, in which the shear stresses are proportional to the shear rate; the flow is laminar; the friction surfaces microgeometry is neglected.

The hydrodynamic pressure field is determined most accurately by employment of the universal equation by Elrod (Elrod, 1981) for the degree of filling of the clearance $\theta$ by lubricant:

$$
\frac{1}{r^{2}} \frac{\partial}{\partial \varphi}\left[\frac{h^{3}}{12 \mu} \beta g \frac{\partial \theta}{\partial \varphi}\right]+\frac{\partial}{\partial z}\left[\frac{h^{3}}{12 \mu} \beta g \frac{\partial \theta}{\partial z}\right]=\frac{\left(\omega_{2}-\omega_{1}\right)}{2} \frac{\partial}{\partial \varphi}(h \theta)+\frac{\left(w_{2}-w_{1}\right)}{2 r} \frac{\partial}{\partial z}(h \theta)+\frac{\partial}{\partial t}(h \theta) .
$$


Where $r$ is the radius of the journal; $\varphi, z$ are the angular and axial coordinates, accordingly (Fig. 1); $h(\varphi, z, t)$ is film thickness; $\mu$ is lubricant viscosity; $\beta$ is lubricant compressibility factor; $\omega_{1}, \omega_{2}$ are the angular velocity of rotation of the bearing and the journal in the inertial coordinate system; $w_{1}, w_{2}$ are forward speed of bearing and journal, accordingly; $t$ is time; $g$ is switching function, $g= \begin{cases}1, & \text { if } \theta \geq 1 ; \\ 0, & \text { if } \theta<1 \text {. }\end{cases}$

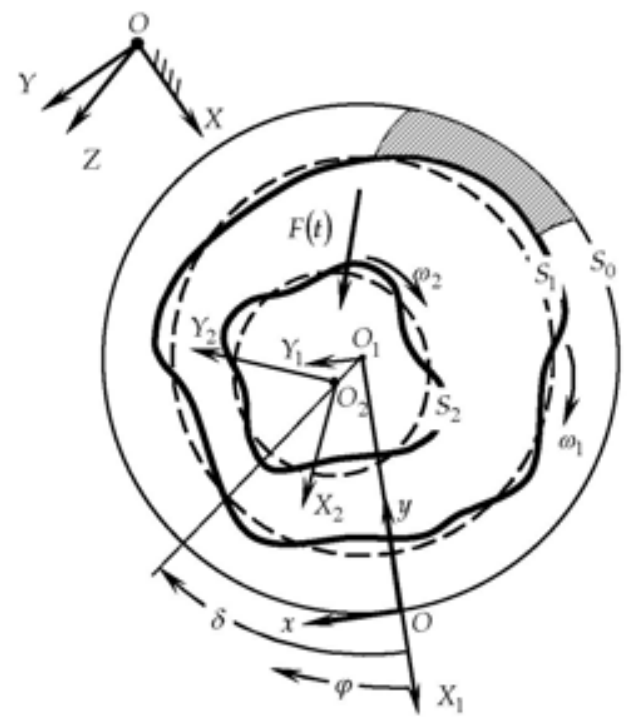

Fig. 1. Cross section bearing

If $\left(\omega_{2}-\omega_{1}\right)=0$, then we get an equation for the tribounit with the forward movement of the journal (piston unit). If $\left(w_{2}-w_{1}\right)=0$, we get the equation for the bearing with a rotational movement of the shaft (radial bearing).

The degree of filling $\theta$ has the double meaning. In the load region $\theta=\rho / \rho_{c}$, where $\rho$ is homogeneous lubricant density; $\rho_{c}$ is the lubricant density if a pressure is equal to the pressure of cavitation $p_{c}$. In the area of cavitation $p=p_{c}, \rho=\rho_{c}$ and $\theta$ determines the mass content of the liquid phase (oil) per a unit of space volume between a journal and a bearing. The relation between hydrodynamic pressure $p(\varphi, z)$ and $\theta(\varphi, z)$ can be written as

$$
p=p_{c}+g \cdot \beta \ln \theta .
$$

The equation (1) allows us to implement the boundary conditions by Jacobson-FlobergaOlsen (JFO), which reflect the conservation law of mass in the lubricating film

$$
\begin{aligned}
& p\left(\varphi_{g}, z\right)=\partial p / \partial \varphi\left(\varphi_{g}, z\right)=p\left(\varphi_{r}, z\right)=p_{a} ; \\
& p(\varphi, z= \pm B / 2)=p_{a} ; p(\varphi, z)=p(\varphi+2 \pi, z),
\end{aligned}
$$

where $\varphi_{g}, \varphi_{r}$ are the corners of the gap and restore of the lubricating film; $B$ is bearing width; $p_{a}$ is atmospheric pressure. 
The conditions of JFO can quite accurately determine the position of the load region of the film. The algorithms of the solution of equation (1), which implement them, are called "a mass conserving cavitation algorithm".

On the other hand the field of hydrodynamic pressures in a thin lubricating film is determined from the generalized Reynolds equation (Prokopiev et al., 2010):

$$
\frac{1}{r^{2}} \frac{\partial}{\partial \varphi}\left[\frac{h^{3}}{12 \mu} \frac{\partial p}{\partial \varphi}\right]+\frac{\partial}{\partial z}\left[\frac{h^{3}}{12 \mu} \frac{\partial p}{\partial z}\right]=\frac{\left(\omega_{2}-\omega_{1}\right)}{2} \frac{\partial h}{\partial \varphi}+\frac{\left(w_{2}-w_{1}\right)}{2 r} \frac{\partial h}{\partial z}+\frac{\partial h}{\partial t} .
$$

The equation (4) was sufficiently widespread in solving problems of dynamics and lubrication of different tribounits.

When integrating the equation (4) in the area $\Omega=(\varphi \in 0,2 \pi ; z \in-B / 2, B / 2)$ mostly often Stieber-Swift boundary conditions are used, which are written as the following restrictions on the function $p(\varphi, z)$ :

$$
p(\varphi, z= \pm B / 2)=p_{a} ; p(\varphi, z)=p(\varphi+2 \pi, z) ; p(\varphi, z) \geq p_{a},
$$

If the sources of the lubricant feeding for the film locate on the friction surfaces, then equations (3) and (5) must be supplemented by

$$
p(\varphi, z)=p_{S} \quad н a(\varphi, z) \in \Omega_{S}, S=1,2 \ldots S^{*},
$$

where $\Omega_{S}$ is the region of lubricant source, where pressure is constant and equal to the supply pressure $p_{S} ; S^{*}$ is the number of sources.

To solve the equations (1) and (3) taking into account relations (3), (5), (6) we use numerical methods, among which variational-difference methods with finite element (FE) models and methods for approximating the finite differences (FDM) are most widely used. These methods are based on finite-difference approximation of differential operators of the boundary task with free boundaries. They can most easily and quickly obtain solutions with sufficient accuracy for bearings with non-ideal geometry. These methods also can take into account the presence of sources of lubricant on the friction surface.

One of the most effective methods of integrating the Reynolds equation are multi-level algorithms, which allows to reduce significantly the calculation time. Equations (1) and (4) are reduced to a system of algebraic equations, which are solved, for example, with the help of Seidel iterative method or by using a modification of the sweep method.

\subsection{Geometry of a heavy-loaded tribounit}

The geometry of the lubricant film influences on hydromechanical characteristics the greatest. Changing the cross-section of a journal and a bearing leads to a change in the lubrication of friction pairs. Thus technological deviations from the desired geometry of friction surfaces or strain can lead to loss of bearing capacity of a tribounit. At the same time in recent years, the interest to profiled tribounits had increased. Such designs can substantially improve the technical characteristics of journal bearings: to increase the carrying capacity while reducing the requirements for materials; to reduce friction losses; to increase the vibration resistance. Therefore, the description of the geometry of the lubricant film is a crucial step in the hydrodynamic calculation. 
Film thickness in the tribounit depends on the position of the journal center, the angle between the direct axis of a journal and a bearing, as well as on the macrogeometrical deviations of the surfaces of tribounits and their possible elastic displacements.

We term the tribounit with a circular cylindrical journal and a bearing as a tribounit with a perfect geometry. In such a tribounit the clearance (film thickness) in any section is equal constant for the central shaft position in the bearing $\left(h^{*}\left(\varphi, Z_{1}\right)=\right.$ const $)$. Where $\varphi, Z_{1}$ are circumferential and axial coordinates.

For a tribounit with non-ideal geometry the function of the clearance isn't equal constant $\left(h^{*}\left(\varphi, Z_{1}\right) \neq\right.$ const $)$. This function takes into account profiles deviations of the journal and the bearing from circular cylindrical forms as a result of wear, manufacturing errors or constructive profiling.

If the tribounit geometry is distorted only in the axial direction, that is $h^{*}\left(Z_{1}\right) \neq$ const, we term it as a tribounit with non-ideal geometry in the axial direction, or a non-cylindrical tribounit. If the tribounit geometry is distorted only in the radial direction, that is $h^{*}(\varphi) \neq$ const, we term it as a tribounit with a non-ideal geometry in the radial direction or a non- radial tribounit (Prokopiev et al., 2010).

For a non- radial tribounit the macro deviations of polar radiuses of the bearing and the journal from the radiuses $r_{i 0}$ of base circles (shown dashed) are denoted by $\Delta_{1}(\varphi), \Delta_{2}(\varphi, t)$. Values $\Delta_{i}$ don't depend on the position $z$ and are considered positive (negative) if radiuses $r_{i 0}$ are increased (decreased). In this case, the geometry of the journal friction surfaces is arbitrary, the film thickness is defined as

$$
h(\varphi, t)=h^{*}(\varphi, t)-e \cos (\varphi-\delta) .
$$

Where $h^{*}(\varphi, t)$ is the film thickness for the central position of the journal, when the displacement of mass centers of the journal in relation to the bearing equals zero $(e(t)=0)$. It is given by

$$
h^{*}(\varphi, t)=\Delta_{0}+\Delta_{1}(\varphi)-\Delta_{2}(\varphi, t), \Delta_{0}=\left(r_{10}-r_{20}\right) .
$$

The function $h^{*}(\varphi, t)$ can be defined by a table of deviations $\Delta_{i}(\varphi, t)$, analytically (functions of the second order) or approximated by series.

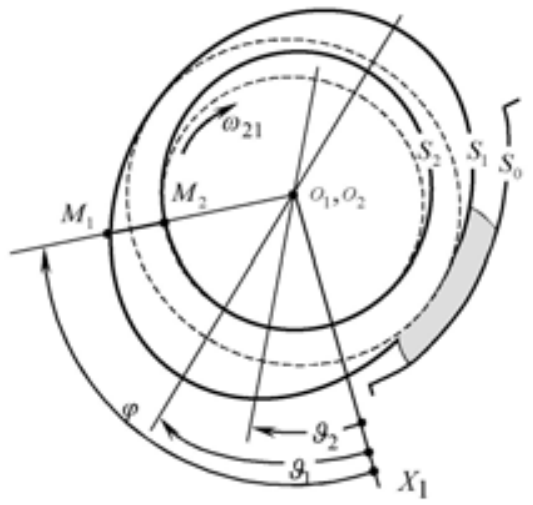

Fig. 2. Scheme of a bearing with the central position of a journal 
If a journal and a bearing have the elementary species of non-roundness (oval), their geometry is conveniently described by ellipses. For example, the oval bearing surface is represented as an ellipse (Fig. 2) and the journal surface is represented as a one-sided oval a half-ellipse.

Using the known formulas of analytic geometry, we represent the surfaces deflection $\Delta_{i}$ of a bearing and a journal from the radiuses of base surfaces $r_{0 i}=b_{i}$ in the following form

$$
\Delta_{i}=b_{i}\left\{v_{i}\left[v_{i}^{2}-\left(v_{i}^{2}-1\right) \cos ^{2}\left(\varphi-\vartheta_{i}\right)\right]^{-0,5}-1\right\},
$$

where the parameter $v_{i}$ is the ratio of high $a_{i}$ to low $b_{i}$ axis of the ellipse, $\vartheta_{i}$ are angles which determine the initial positions of the ovals.

Due to fixing of the polar axis $O_{1} X_{1}$ on the bearing, the angle $\vartheta_{1}$ doesn't depend on the time, and the angle $\vartheta_{20}$, which determines the location of the major axis of the journal elliptic surface with $t=t_{0}$, is associated with a relative angular velocity $\omega_{21}$ by the following relation

$$
\vartheta_{2}(t)=\vartheta_{20}+\int_{t_{0}}^{t} \omega_{21} d t
$$

In an one-sided oval of a journal equation (9) is applied in the field $\left(\pi / 2+\vartheta_{2}\right) \leq \varphi \leq\left(3 / 2 \pi+\vartheta_{2}\right)$, but off it $\Delta_{2}=0$.

If the macro deviations $\Delta_{1}(\varphi), \Delta_{2}\left(\gamma_{2}\right)$ of journal and bearing radiuses $r_{i}(\varphi)$ from the base circles radiuses $r_{i 0}$ are approximated by truncated Fourier series, then they can be represented as (Prokopiev et al., 2010):

$$
\Delta_{i}(\psi)=\tau_{i 0}+\tau_{i} \sin \left(k_{i} \psi+\alpha_{i}\right),
$$

where $i=1$ for a bearing, $i=2$ for a journal; $\psi=\varphi$ if $i=1, \psi=\gamma_{2}=\varphi+\vartheta_{1}-\vartheta_{2}$ if $i=2$; $\vartheta_{2}=\int_{0}^{t} \omega_{21}(t) d t ; k_{i}$ is a harmonic number; $\tau_{i}, \alpha_{i}$ are the amplitude and phase of the $k$-th harmonic; $\tau_{i 0}$ is a permanent member of the Fourier series, which is defined by

$$
\tau_{i 0}=\frac{1}{2 \pi} \int_{0}^{2 \pi} \Delta_{i}(\psi) d \varphi .
$$

For elementary types of non-roundness (oval $(k=2)$; a cut with three $(k=3)$ or four $(k=4)$ vertices of the profile) $\tau_{i 0}=0$.

The thickness of the lubricant film, which is limited by a bearing and a journal having elementary types of non-roundness, after substituting (12) in (7), is given by

$$
h(\varphi, t)=\Delta_{0}+\tau_{1} \sin \left(k_{1} \varphi+\alpha_{1}\right)-\tau_{2} \sin \left(k_{2} \gamma_{2}+\alpha_{2}\right)-e \cos (\varphi-\delta) .
$$

For tribounits with geometry deviations from the basic cylindrical surfaces in the axial direction the film thickness at the central position of the journal in an arbitrary cross-section $Z_{1}$ is written by the expression 


$$
h^{*}\left(Z_{1}\right)=\Delta_{0}+\Delta_{1}\left(Z_{1}\right)-\Delta_{2}\left(Z_{1}\right) .
$$

Where $\Delta_{i}\left(Z_{1}\right), i=1,2$ are the deviations of generating lines of bearing surfaces and the journal surfaces from the line (positive deviation is in the direction of increasing radius).

Then, taking into account the expressions (8) and (14) we can write the general formula for a lubricant film thickness with the central position of the journal in the bearings with nonideal geometry as

$$
h^{*}\left(\varphi, Z_{1}, t\right)=\Delta_{0}+\Delta_{1}(\varphi)-\Delta_{2}(\varphi, t)+\Delta_{1}\left(Z_{1}\right)-\Delta_{2}\left(Z_{1}\right) .
$$

A barreling, a saddle and a taper are the typical macro deviations of a journal and a bearing from a cylindrical shape (Fig. 3).

a)

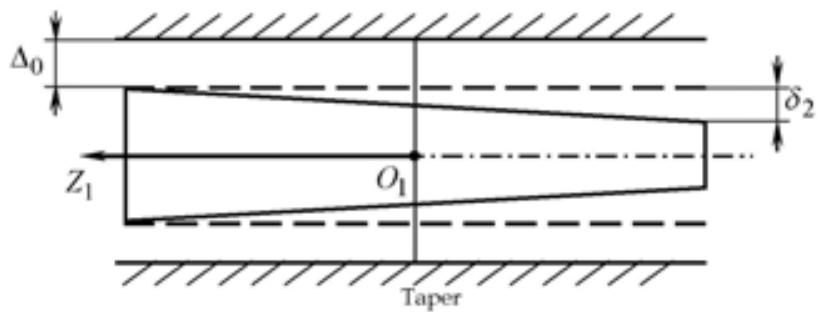

b)

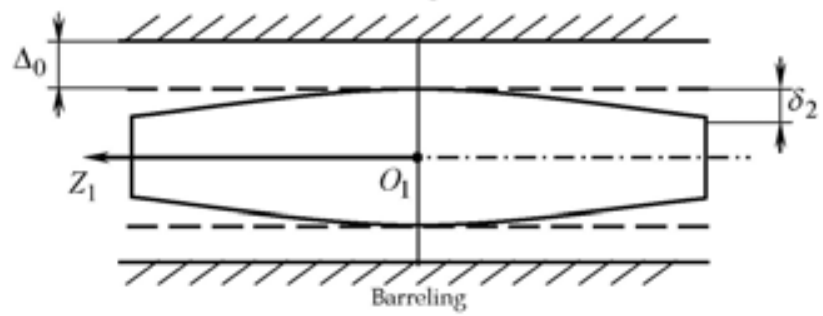

c)

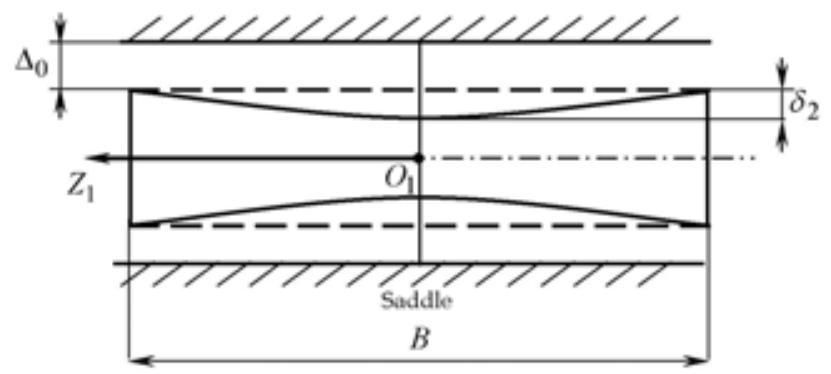

Fig. 3. Types of non-cylindrical journals

The non-cylindrical shapes of the bearing and the journal in the axial direction are defined by the maximum deviations $\delta_{1}$ and $\delta_{2}$ of a profile from the ideal cylindrical profile and are described by the corresponding approximating curve. Then the film thickness at the central position of the journal (Prokopiev et al., 2010) is given by

$$
h^{*}\left(Z_{1}\right)=\Delta_{0}+k_{1} Z_{1}^{l_{1}}+k_{2} Z_{1}^{l_{2}},
$$


where $k_{i}$ defines the deviation of the approximating curve per unit of the width of the bearing, the degree of the parabola is accepted: $l_{i}=1$ for the conical journals; $l_{i}=2$ for barrel and saddle journals.

For the circular cylindrical bearing for $\Delta_{i}=0$ the film thickness is determined by the wellknown formula:

$$
\bar{h}(\varphi, \bar{t})=1-\chi \cos (\varphi-\delta) .
$$

For the circular cylindrical journal its rotation axis is parallel to the axis $O_{1} Z_{1}$. In practice, the axis of the journal may be not parallel to the axis of the bearing, so there is a so-called "skewness". These deviations may be as due to technological factors (the inaccuracy of manufacturing during the production and repair) as to working conditions (wear, bending of shafts, etc.).

Position of the journal, which is regarded as a rigid body, in this case you can specify by two coordinates $e, \delta$ of the journal center $\mathrm{O}_{2}$ and by three angles $\left(\gamma, \varepsilon, \theta_{2}\right)$. Angle $\gamma$ is skewness of journal axis; $\varepsilon$ is the deviation angle of skewness plane from the base coordinate plane; $\theta_{2}$ is the rotation angle of the journal on its own axis $\mathrm{O}_{2} \mathrm{Z}_{2}$.

When journal axis is skewed the film thickness at a random cross-section $Z_{1 i}$ of the bearing depends on the eccentricity $e_{i}$ and the angle $\delta_{i}$ for this cross-section

$$
h\left(\varphi, Z_{1 i}, t\right)=h^{*}\left(\varphi, Z_{1 i}\right)-e_{i} \cos \left(\varphi-\delta_{i}\right),
$$

where $h^{*}\left(\varphi, Z_{1 i}\right)$ is the film thickness with the central journal position in $i$-th cross -section. We term the $\operatorname{tg} \gamma=2 s / B$, where $s$ is the distance between the geometric centers of the journal and the bearing at the ends of the tribounit; $B$ is the width of the tribounit. The expression for the lubricant film thickness, taking into account the skewness, is written in the form

$$
h\left(\varphi, Z_{1}, t\right)=h^{*}\left(\varphi, Z_{1}\right)-e \cos (\varphi-\delta)-Z_{1} \cdot \frac{2 s}{B} \cos (\varphi-\varepsilon) .
$$

It should be also taken into account that the bearing surfaces are deformed under the action of hydrodynamic pressures. The value $\Delta(p)$ is the radial elastic displacement of the bearing sliding surface under the action of hydrodynamic pressure $p$ in the lubricant film. Function $\Delta(p)$ is defined in the process of calculating of the bearing strain (for a "hard" bearing $\Delta(p)=0)$ and is written in the form of a component in the equation for the lubricant film thickness.

Thus, the film thickness, taking into account the arbitrary geometry of friction surfaces of a journal and a bearing, the skewness of the journal and elastic displacements of the bearing, is determined by the equation:

$$
h\left(\varphi, Z_{1}, t\right)=h^{*}\left(\varphi, Z_{1}\right)-e \cos (\varphi-\delta)-Z_{1} \cdot 2 s / B \cdot \cos (\varphi-\varepsilon)+\Delta(p)
$$

where $h^{*}\left(\varphi, Z_{1}\right)$ is the film thickness with the central position of the journal in the bearing with non-ideal geometry; $e(t)$ is displacement of journal mass centers in relation to the bearing; $\varepsilon(t)$ - an angle that takes into account the skewness of axes of a bearing and a journal. The values $e(t), \delta(t), \varepsilon(t)$ are determined by solving the equations of motion. 


\subsection{The calculation of thermal processes}

The theory of thermal processes in the heavy-loaded tribounit of fluid friction is based on a generalized equation of energy (heat transmission) for a thin film of viscous incompressible fluid, which is between two moving surfaces $S_{1}$ and $S_{2}$. If we assume a low thermal conductivity in the direction of the coordinate axes $O x z$ (the axis $O y$ is normal to the surface $\left.S_{1}\right)$ (Fig. 1), the temperature distribution $T(x, y, z, t)$ in the lubricating film will be described by the equation (Prokopiev\&Karavayev, 2003)

$$
\rho c_{0} \frac{\partial T}{\partial t}+\rho c_{0}\left(V_{x} \frac{\partial T}{\partial x}+V_{y} \frac{\partial T}{\partial y}+V_{z} \frac{\partial T}{\partial z}\right)-\lambda_{0} \frac{\partial^{2} T}{\partial y^{2}}=\text { Д. }
$$

Where $\rho$ is density; $c_{0}, \lambda_{0}$ are specific heat capacity and thermal conductivity of lubricant (usually taken as constant); $t$ is the time; Д is the dissipation function, which is defined for non-Newtonian fluid by the approximate expression

$$
\text { Д } \approx \mu^{*} I_{2} .
$$

The three approaches to the integration of the equation (21) (thermohydrodynamic (nonisothermal), adiabatic, isothermal) can be used, depending on the assumptions which are used about the temperature distribution in a thin lubricating film.

When thermohydrodynamic approach is applied the temperature will change in all directions, including across the oil film. In this case, the boundary conditions are stated quite simply and are the most adequate to the real thermal processes. With this approach, we get information about the local properties of the temperature field of lubricating film: a temperature distribution $T(x, y, z, t)$; maximum temperature $T_{\max }$, instantaneous average temperature $T_{a v}(t)$; zones of elevated temperatures.

If adiabatic approach is applied the change of the temperature across the oil film (along the axis $O y$ ) is ignored, the journal and the bearing are assumed ideal thermal insulators. We introduce a computational averaged over the width of the bearing temperature $T^{*}=T^{*}(x, t)$. We substitute it into the equation (21) and receive a differential equation for the temperature distribution along the coordinate $x$. Since in this case the heat transfer to the journal and the bearing is not taken into account, the calculated temperatures are too high. It reduces the accuracy of the results.

The isothermal approach assumes that the calculated current temperature $T_{c}=T_{c}(t)$ is the same at all points of the lubricant film. This temperature is a highly inertial parameter and it is determined by solving the heat balance equation

$$
A_{N}^{*}(t)=A_{Q}^{*}(t) \text {. }
$$

This equation reflects the equality of the average values of the heat $A_{N}^{*}$, which is dissipated in the lubricating film, and the average values of the heat $A_{Q}^{*}$, which is drained by lubricant into the ends of the tribounit during the loading cycle.

The accurate definition of the current temperature can be performed: at each time step of the calculation; once per a cycle of loading the tribounit, at each time step of the calculation taking into account the thermal interaction between the lubricant film with a journal, with a bearing and a lubrication groove. 


\subsection{The equations of heavy-loaded bearing dynamics}

To study the dynamics of bearings of liquid friction the motion of the journal on the lubricant film in the bearing is usually considered (Fig. 4). In the coordinates space OXYZ the movement of the journal, which rotates with the relative angular velocity and the angular acceleration, taking into account the axle skewness of a journal and a bearing, is described by approximate differential equations

$$
\tilde{m} \ddot{U}(t)=\tilde{F}(t)+\tilde{R}(U, \dot{U}) \text {. }
$$

Where $\tilde{m}$ is the matrix of inertia of the journal: $\left\{\tilde{m}_{i i}\right\}=\left\{m, m, m, J_{X}, J_{Y}, J_{Z}\right\}, m, J_{X}, J_{Y}, J_{Z}$ are mass and moments of inertia of the journal, $\tilde{m}_{i j \neq i}=0, i=1, \ldots, 6, j=1, \ldots, 6$;

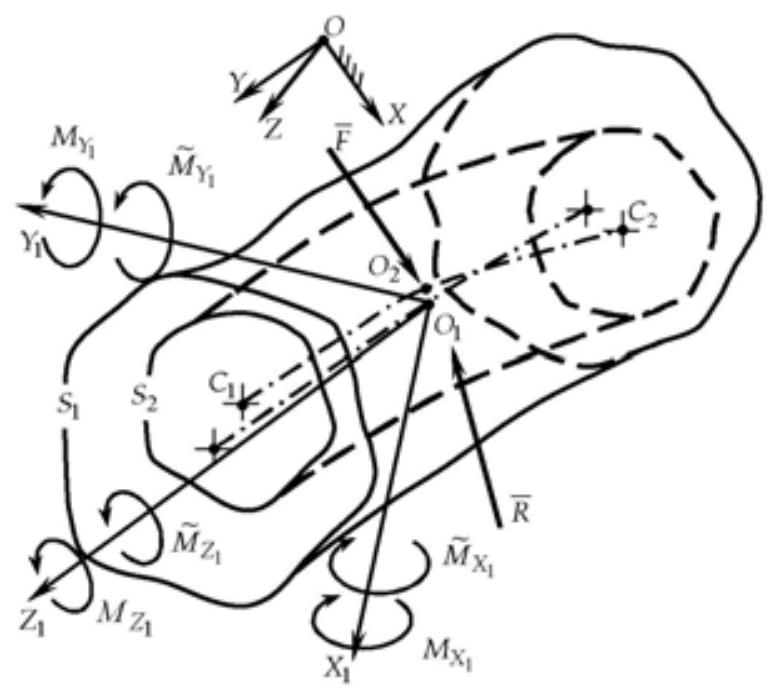

Fig. 4. Scheme of a heavy-loaded bearing with arbitrary geometry of the lubricant film

$U(t)=\left\{X, Y, Z, \gamma_{X}, \gamma_{Y}, \gamma_{Z}\right\}$ is the vector of generalized coordinates of the journal centre; $\tilde{F}(t)=\left\{F_{X}, F_{Y}, F_{Z}, M_{X}, M_{Y}, M_{Z}\right\}$ is the vector of known loads on the journal, presented by the power $\bar{F}$ with its projections $F_{X}, F_{Y}, F_{Z}$ on the axes of the coordinate system OXYZ and a moment of forces $\bar{M}$ with its projections $M_{X}, M_{Y}, M_{Z} ; \tilde{R}(U, \dot{U})=\left\{R_{X}, R_{Y}, R_{Z}, \mathrm{M}_{X}, \mathrm{M}_{Y}, \mathrm{M}_{Z}\right\}$ is the vector of loads due to the hydrodynamic pressure in the lubricant film. The time derivatives are denoted by points. The forces of friction and weight, as well as gyroscopic moments of the rotating journal are considerably less than other loads, so they aren't taken into account in the equations of motion.

For the dynamics of radial bearings of ICE the level of loads $\bar{F}$ acting on the journal is higher than its own inertial forces. The system of equations of motion (24) in this case is rewritten as

$$
\tilde{F}(t)+\tilde{R}(U, \dot{U})=0
$$


Projections of linear and angular positions and velocities and loads $\bar{F}, \bar{M}, \bar{R}, \overline{\mathrm{M}}$ onto the axis $\mathrm{OZ}$ are excluded from the employed vectors.

In the case of planar motion of a journal on the lubricant film the solution to the problem of the dynamics of the radial bearings can be obtained more easily. The skewness of axes of a journal and a bearing are neglected:

$$
\gamma_{X}=\gamma_{Y}=\gamma_{Z}=M_{X}=M_{Y}=M_{Z}=0, \tilde{M}_{X}=\tilde{M}_{Y}=\tilde{M}_{Z}=0 .
$$

The vectors of coordinates, velocities and loads include only their projections on the axes OX,OY.

The solution of the systems of equations of motion (24) or (25) can be found only numerically, because the loads, which are caused by the hydrodynamic pressure, are determined by the repeated numerical solution of the differential equations by Elrod (1) or by Reynolds (4). If we discretize the system of equations of motion over time, then the decision when passing to the next time step can be obtained by using the explicit or implicit method of calculation. In an explicit scheme the unknowns are the pressure and the coordinates of the journal center, in an implicit scheme the unknowns are the pressure and the rate of position change of the journal. However, the implementation of explicit schemes of integrating the motion equations is sensitive to the accumulation of rounding errors. Therefore, implicit schemes for integrating the equations of motion over time are realized in several studies, which are dedicated to the dynamics of heavy-loaded tribounits.

The most common methods for solving equations of motion of type (24) are: Newton's method, Runge-Kutta's method with Merson's modification, the modified method of linear acceleration (Wilson's method), the method of non-central third-order differences (method by Habolt). To solve the system of the form (25) it is expedient to use special techniques, which are adapted to the systems of "stiff" differential equations (method based on the use of differentiation backward formulas (DBF) of the first- and second-order, method by Fowler, Wharton and others). The standard procedure for solving differential equations (25), which are unsolved relatively to derivatives, consists in the formal integration of the equations $\dot{U}=f(U, t)$ and determining derivatives with the help of Newton method.

When the character of applied loads is periodical the initial values of variables $U$ and their derivatives $\dot{U}$ can be set arbitrarily. With that the integration continues until the time when the values $U$ and $\dot{U}$, which are separated by a period $t_{c}$ of load changes, will not be repeated.

Ability to use a particular method of integration depends on the type of a tribounit, the character of acting loads and the possibility to set an initial approximation for the successful solution of (24) or (25). Currently, universal methods for solving the dynamics of heavyloaded tribounits are not designed. The result of calculating the dynamics of heavy-loaded bearings is a trajectory of mass center of the journal, as well as hydro-mechanical characteristics of tribounits.

The construction of the heavy-loaded tribounit is evaluated by parameters of the calculated trajectory and interconnected hydro-mechanical characteristics (HMCh). There is the lowest and average per cycle of loading values of: the lubricant film thickness $\inf h_{\min }, h_{\min }^{*}, \mu m$; the hydrodynamic pressure in the lubricant film $\sup p_{\max }, p_{\max }^{*}, M P a$; the unit load $f_{\max }$, $f_{\max }^{*}, M P a$; the relative total length of the regions $\alpha_{h_{\partial о}}$, where the values of $h_{\text {min }}$ less than allowable values $h_{\text {до }}, \%$; the relative total length of the regions $\alpha_{p_{\text {до }}}$, where the values of $p_{\max }$ greater than allowable values $p_{\text {доn }}, \%$; mean-value losses due to friction $N^{*}, \mathrm{~W}$, the 
leakage of lubrication in the bearing ends $Q^{*}, M^{3} / s$ and temperature of the lubricant film $T,{ }^{\circ} \mathrm{C}$.

\section{Lubrication with non-Newtonian and multiphase fluids}

The development of technology is inextricably linked with the improvement of lubricants, which today remain an important factor that ensures the reliability of machines. Currently, for lubrication of tribounits of ICE multigrade oils are widely used, rheological behavior of which does not comply with the law of Newton-Stokes equations on a linear relationship between shear stress and shear rate (Whilkinson, 1964):

$$
\tau=\mu \cdot \dot{\gamma},
$$

where $\tau$ - shear stress; $\mu$ - dynamic viscosity, which is a function of temperature $T$ and pressure $p$ (Newtonian viscosity); $\dot{\gamma}$ - shear rate, $\dot{\gamma}=\sqrt{I_{2}} ; I_{2}$ - second invariant of shear rate $I_{2} \approx\left(\partial V_{x} / \partial y\right)^{2}+\left(\partial V_{z} / \partial y\right)^{2}, V_{x}, V_{y}, V_{z}$ - velocity component of the elementary volume lubrication, which is located between the two surfaces.

Particularly, the viscosity depends not only on the temperature and pressure, but also on the shear rate in a thin lubricating film separating the surfaces of friction pairs. These oils are called non-Newtonian.

Theoretical studies of the dynamics of friction pairs, which take into account nonNewtonian behavior of lubricant, are based on the modification of the equations for determining the field of hydrodynamic pressures by using different rheological models. One classification of a rheological model is shown in Fig. 5.

In general, non-Newtonian behavior includes any anomalies observed in the flow of fluid. In particular, the presence of viscous polymer additives in oils leads to a change in their properties. Oils with additives can be characterized as structurally viscous and viscoelastic Viscoelastic fluids are those exhibiting both elastic recovery of form and viscous flow. There are various models of viscoelastic fluids, among which the best known model is the Maxwell $\tau+\lambda \frac{\partial \tau}{\partial t}=\mu^{*} \dot{\gamma}$. Here $\lambda$ - relaxation time, characterizing the delay of shear stress changes in respect to changes of shear rates; $\mu^{*}(T, p, \dot{\gamma})$ - dynamic viscosity (non-Newtonian viscosity). In this case, the liquid is called the Maxwell (Maxwell viscoelastic liquid).

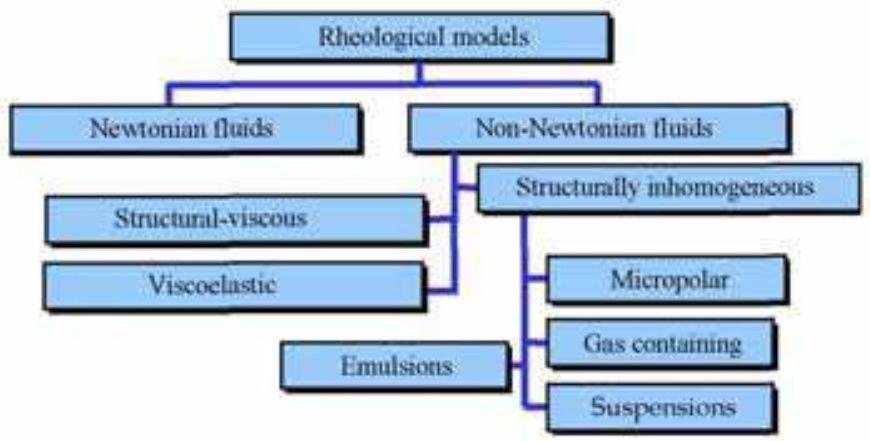

Fig. 5. Classification of rheological models of lubricating fluids 
It is assumed that the viscoelastic properties of thickened oils have a positive impact on the operation of sliding bearings, help to increase the thickness of the lubricant film. Qualitative influence of viscoelastic properties (relaxation time) of the lubricant is reflected in Fig. 6.

With the increase of the relaxation time of lubrication, the mean-value of the minimum lubricating film thickness and power loss due to friction increase. It is seen that the character of the dependence is the same, but the values are shifted back to the rotation angle of the crankshaft.
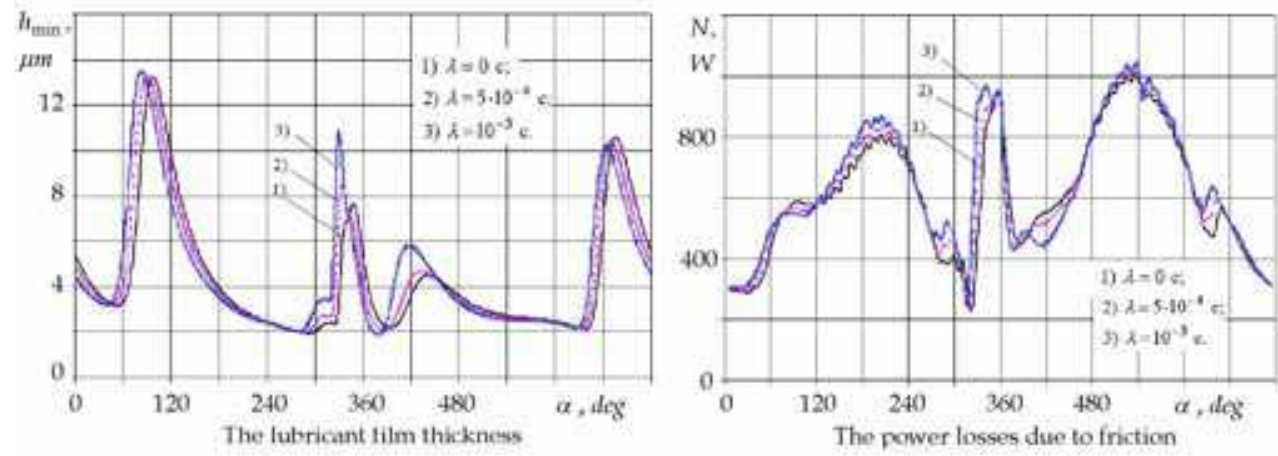

Fig. 6. The dependence of the characteristics from the angle of rotation of crankshaft

Structural-viscous oils have the ability to temporarily reduce the viscosity during the shear, so they are called "energy saving", because they help to reduce power losses due to friction in internal combustion engines and, consequently, fuel consumption (according to various estimates by $2-5 \%)$.

The most well-known mathematical model describing the behavior of the structural-viscous oils, is a power law of Ostwald-Weyl, according to which the dependence of viscosity versus shear rate is defined as (Whilkinson, 1964)

$$
\mu^{*}=k \dot{\gamma}^{n-1} .
$$

Where $k$ - measure the fluid consistency; $n$ - index characterizing the degree of nonNewtonian behavior.

Gecim suggested the dependence of viscosity on the second invariant of shear rate, which is based on the concept of the first $\mu_{1}(T)$ and the second $\mu_{2}(T)$ Newtonian viscosity, the parameter $K_{c}(T)$, characterizing the shear stability of lubricants (Gecim, 1990):

$$
\mu^{*}(\dot{\gamma})=\mu_{1} \frac{K_{c}+\mu_{2} \cdot \dot{\gamma}}{K_{c}+\mu_{1} \cdot \dot{\gamma}} .
$$

The higher $K_{c}$, the higher is the stability of the liquid with respect to the shift. At low shear viscosity value corresponds to the $\mu_{1}$, with increasing shear rate the viscosity tends to $\mu_{2}$ (Fig. 7). Experimental studies have established that multigrade oils of the same viscosity grade of SAE may have different shear stability.

The application of structural-viscous oils, along with a reduction of power losses to friction leads to a decrease in the lubricating film thickness, temperature and to the increase of lubrication flow rate. 


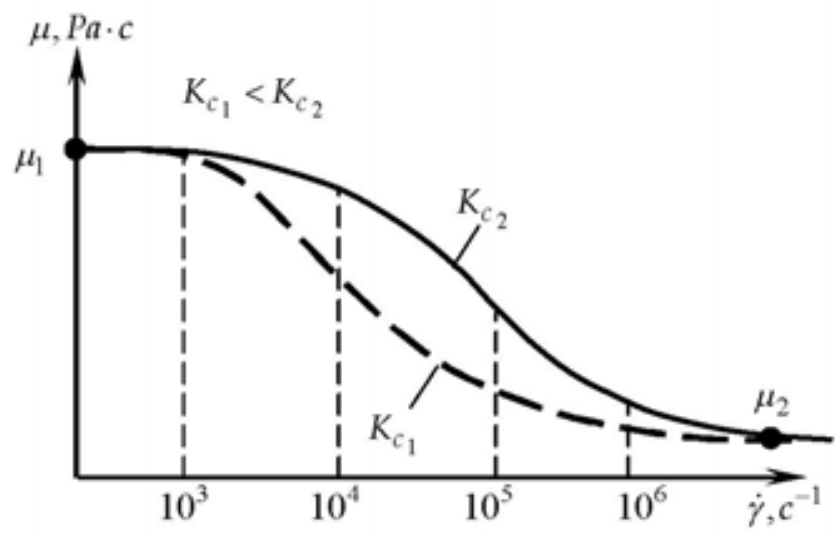

Fig. 7. Fundamental character of the non-Newtonian oils viscosity

Comparative results of the calculation of hydro-mechanical characteristics of the connecting rod bearing for the dependence of oil viscosity versus shear rate and without it are presented in Table. 1 and Fig. 8.

All results were founded for connecting-rod bearing of engine type ЧН 13/15 (Co ltd. "ChTZ-URALTRAC") with follow parameters: rotating speed $219.91 \mathrm{c}^{-1}$; length $0.033 \mathrm{~m}$; journal radius $0.0475 \mathrm{~m}$; radial clearance $51.5 \mu \mathrm{m}$.

The results indicate that the application of structural-viscous oils leads to a reduction of power losses due to friction in the range $15-20 \%$. Consumption of lubricant through the bearing increases, the mean-value of the temperature decreases by $2-3^{\circ} \mathrm{C}$. However, there is a decrease in the minimum lubricating film thickness by an average of $14-20 \%$.

This fact confirms the view that the use of low-viscosity oil at high temperature and shear rate is justified only if it is allowed by the engine design, in particular, of crankshaft bearings.

\begin{tabular}{|c|c|c|c|c|c|c|c|}
\hline $\begin{array}{c}\text { Hydromechanical } \\
\text { characteristics }\end{array}$ & $\begin{array}{c}N^{*}, \\
W\end{array}$ & $\begin{array}{c}T^{\circ} C^{\prime} \\
\text { Newtonian fluid }\end{array}$ & $\begin{array}{c}Q_{B}^{*} \\
l / s\end{array}$ & $\begin{array}{c}h_{\min }^{*} \\
\mu m\end{array}$ & $\begin{array}{c}\sup p_{\max }{ }^{\prime} \\
M P a\end{array}$ & $\begin{array}{c}\inf h_{\min }{ }^{\prime} \\
\mu m\end{array}$ & $\begin{array}{c}\alpha^{*}, \\
\%\end{array}$ \\
\hline $\begin{array}{c}\text { Structural-viscous } \\
\text { liquid (28) }\end{array}$ & 518,4 & 105,9 & 0,02345 & 4,416 & 280,3 & 1,93 & 0 \\
\hline $\begin{array}{c}\text { Structural-viscous } \\
\text { liquid (29) }\end{array}$ & 539,0 & 103,4 & 0,0246 & 3,789 & 307,8 & 1,66 & 11,9 \\
\hline
\end{tabular}

Table 1. The results of the calculation of HMCh of the connecting rod bearing

In recent years, the oil, which has in its composition the so-called friction modifiers, for example, particles of molybdenum, is widespread. These additives are introduced into the base oil to improve its antiwear and extreme pressure properties to reduce friction and wear under semifluid and boundary lubrication regimes.

Oils with such additives are called "micropolar". They represent a mixture of randomly oriented micro-particles (molecules), suspended in a viscous fluid and having its own rotary motion. 

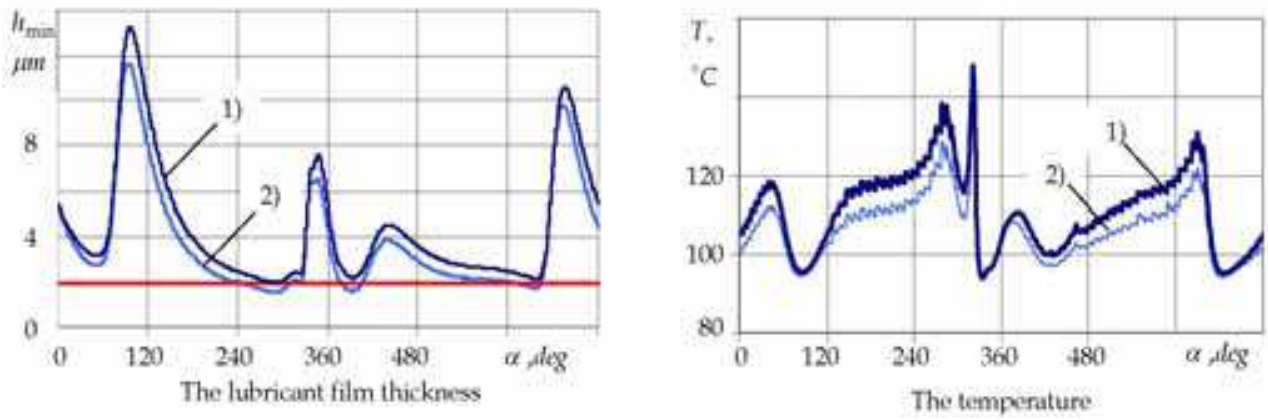

Fig. 8. The dependence of the hydromechanical characteristics from the rotation angle of crankshaft: 1) Newtonian fluid, and 2) the structural-viscous liquid (28)

Micropolar fluid along with the viscosity $\mu$ additionally characterized by two physical constants $\mu_{1}, \ell$. Parameter $\mu_{1}$, called the coefficient of eddy viscosity, takes into account the resistance to micro-rotation of particles. Length parameter $\ell$ characterizes the size of microparticles or molecular lubricant. With the help of the coefficient $\mu_{1}$ and the parameter $\ell$ you can calculate the so-called micropolar parameters

$$
N=\left(\frac{\mu_{1}}{2 \mu+\mu_{1}}\right)^{1 / 2}, L=\frac{h_{0}}{\ell},
$$

where $h_{0}$ - characteristic film thickness.

The presence of micro-particles in the lubricant leads to an increase in the resultant shear stress in the lubricating film. The calculations of heavy-loaded bearings using micropolar fluid theory suggest that this phenomenon significantly affects the HMCh of a bearing, in particular, leads to an increase of lubricating film thickness. The results of the calculation of the connecting rod bearing, taking into account the structural heterogeneity of lubricants (based on the model of micropolar fluids with the parameters $L=10, N^{2}=0,5$ ) are reflected in Fig. 9 and Table. 2.

\begin{tabular}{|c|c|c|c|c|c|c|c|}
\hline $\begin{array}{c}\text { Hydromechanical } \\
\text { characteristics }\end{array}$ & $\begin{array}{c}N^{*}, \\
W\end{array}$ & $\begin{array}{c}T, \\
{ }^{\circ} C\end{array}$ & $\begin{array}{c}Q_{B}^{*}, \\
l / s\end{array}$ & $\begin{array}{c}h_{\min }^{*} \\
\mu m\end{array}$ & $\begin{array}{c}\sup p_{\max }, \\
M P a\end{array}$ & $\begin{array}{c}\inf h_{\min }{ }^{\prime} \\
\mu m\end{array}$ & $\begin{array}{c}\alpha^{*}, \\
\%\end{array}$ \\
\hline Newtonian fluid & 610,5 & 105,9 & 0,02355 & 4,416 & 280,3 & 1,93 & 0 \\
\hline $\begin{array}{c}\text { Structurally } \\
\text { heterogeneous } \\
\text { fluid (30) }\end{array}$ & 727,4 & 110,6 & 0,0215 & 5,84 & 237,9 & 2,9 & 0 \\
\hline
\end{tabular}

Table 2. The results of the calculation of hydro-mechanical characteristics of the connecting rod bearing, taking into account the structural heterogeneity of lubrication

It is obvious, that the results will prove valuable for practice, only in case of experimental determination of the value of the micropolarity parameters $N$ and $L$. Further studies of the authors are focused on the experimental basis of these values for modern thickened oils. 
The calculation of the structural heterogeneity of the lubricant is a very complicated mathematical problem, since it is necessary to take into account many factors: the speed and shape of particles, their distribution, elasticity, etc.
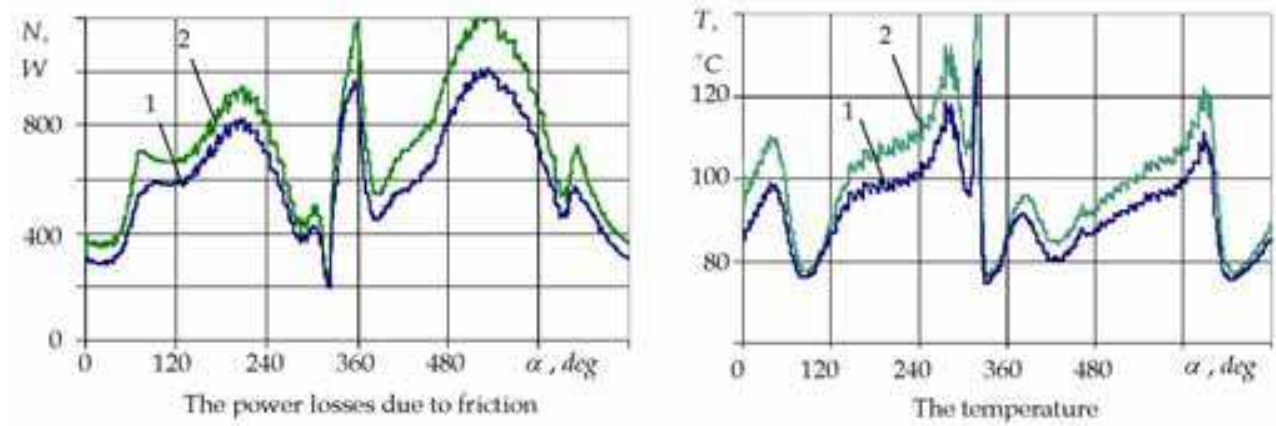

Fig. 9. Dependence of the hydromechanical characteristics from the rotation angle of the crankshaft: 1 - Newtonian fluid; 2 - structurally heterogeneous fluid (30)

Sometimes simplified dependence is used. For example it is assumed that the viscosity of suspensions depends on the concentration volume of solid particles, which may be the wear products, external contaminants or finely divided special additives. In this case, the viscosity of the lubricant is sufficiently well described by the Einstein formula:

$$
\mu=\mu^{*}(1+\xi \cdot \varphi) .
$$

Where $\xi$ - shape factor of particles, for asymmetric particles $\xi \geq 2,5$.

Separate scientific problem is the availability of records in the lubricant gas component. Experimental studies have shown that the engine lubrication system always contains air dissolved in the form of gas bubbles. The proportion of bubbles in the total amount of oil may reach $30 \%$.

The viscosity of gassy oils can be calculated with a sufficient degree of accuracy with the help of the formula:

$$
\mu=\mu^{*}(1-\delta) .
$$

Where the coefficient $\delta=V_{\Gamma} / V_{M}$ is equal to the ratio of the volume fraction of gas $V_{\Gamma}$ in the bubble mixture to the volume fraction of pure oil $V_{M}$ at temperature $T$.

When you select computer models you must take into account not only the working conditions, regime and geometric characteristics of tribounits under consideration, but also features of rheological behavior of used lubricants.

At present, as a result of parallel and interdependent modifications of ICE and production technologies of motor oils, the most loaded sliding bearings of an engine work at the minimum design film thickness of about 1 micron in the steady state and less - at low frequencies of crankshaft rotation, that is with film thicknesses comparable to twice the height of surface roughness of tribounits. In this case the life of one and the same friction unit can vary in 3 ... 5 times when using different motor oils, and be by orders of magnitude greater than the resource when using other grease lubricants at the same bulk rheological properties. 
Based on experimental and theoretical studies it can be argued that under changing conditions of friction a repeated change of mechanisms of friction and wear occurs, in which the key role is played by the change of rheological properties of lubricants, depending on the thickness of the film, the contact pressure, surface roughness and the individual properties of the lubricant. Thus, there is a need for the computational models depending on the rheological properties of lubricating oil on the factors related to the availability, quantity and structure of the antifriction and antiwear additives and lubricants interaction with the surfaces of the friction.

One model describing the dependence of viscosity of lubricant on thickness is proved in (Mukhortov et al., 2010) and has the following form:

$$
\mu_{i}=\mu_{0}+\mu_{S} \exp \left(-\frac{h_{i}}{l_{h}}\right)
$$

where $l_{h}$ - characteristic parameter having the dimension of length, which value is specific for each combination of lubricant and the solid surface; $\mu_{S}$ - parameter having the meaning of the conditional values of the viscosity at infinitely small distance from the bounding surface; $\mu 0$ - viscosity in entirety.

The impact of the availability of a highly viscous boundary film on the friction surfaces on the HMCh of the rod bearing is illustrated in Fig.10 and Table. 3.

In the hydrodynamic friction regime the presence of adsorption films leads to an increase in the minimum lubricating film thickness by $40-45 \%$, the temperature at $6-7 \%$, the maximum hydrodynamic pressure by $4-5 \%$.

\begin{tabular}{|c|c|c|c|c|c|}
\hline $\begin{array}{c}\text { Hydromechanical } \\
\text { characteristics }\end{array}$ & $\begin{array}{c}N^{*}, \\
W^{\prime}\end{array}$ & $\begin{array}{c}{ }^{\circ} C^{\prime} \\
\text { numerical value }\end{array}$ & $\begin{array}{c}h_{\min }^{*} \\
\mu m\end{array}$ & $\begin{array}{c}\sup p_{\max }{ }^{\prime} \\
M P a\end{array}$ & $\begin{array}{c}\inf h_{\min } \\
\mu m\end{array}$ \\
\hline $\left.681,5^{1)} 2\right)$ & 105,9 & 4,416 & 280,3 & 1,93 \\
& 113,3 & 5,665 & 294,9 & 3,59 \\
\hline
\end{tabular}

1 - Newtonian fluid; 2 - taking into account the highly viscous boundary film.

Table 3. The results of the calculation of hydro-mechanical characteristics of the connecting rod bearing in the light of high-viscosity boundary film lubrication
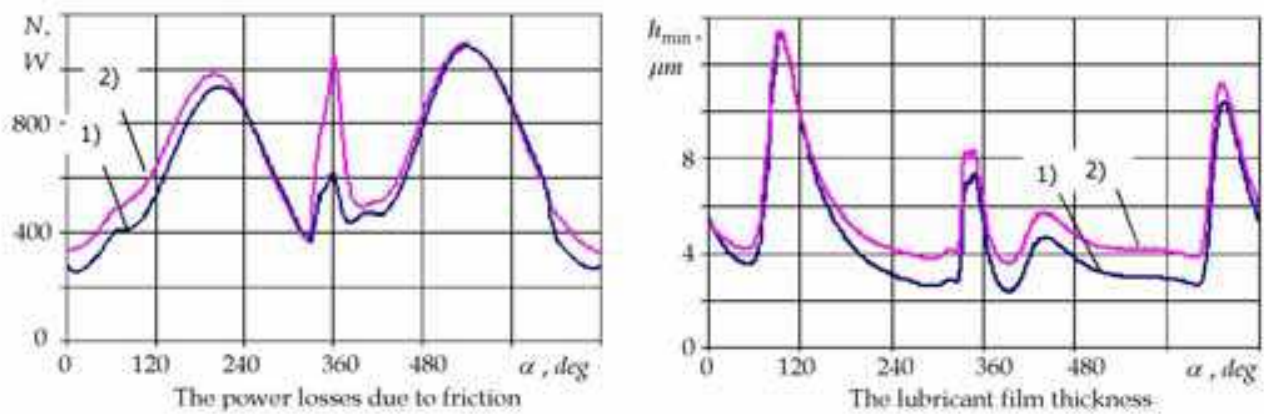

Fig. 10. The dependence of the hydromechanical characteristics from the rotation angle of crankshaft: 1 - Newtonian fluid, 2 - with the boundary layer (33) 
These models should be used in accordance with the terms of the friction pairs. In this case, the use of non-Newtonian models of lubricants does not exclude taking into account the dependence of oil viscosity on temperature and pressure in the lubricating film of friction pairs (Prokopiev V. et al., 2010):

$$
\mu(T)=C_{1} \cdot \exp \left(C_{2} /\left(T+C_{3}\right)\right),
$$

where $C_{1}, C_{2}, C_{3}$ - constants, which are the empirical characteristics of the lubricant.

The coefficients $C_{i}$ are calculated using the formula following from the dependence (34):

$$
\begin{gathered}
C_{3}=\frac{-\left[T_{1}\left(T_{3}-T_{2}\right) \ln \left(\frac{\mu_{1}}{\mu_{2}}\right)-T_{3}\left(T_{2}-T_{1}\right) \ln \left(\frac{\mu_{2}}{\mu_{3}}\right)\right]}{\left[\left(T_{3}-T_{2}\right) \ln \left(\frac{\mu_{1}}{\mu_{2}}\right)-\left(T_{2}-T_{1}\right) \ln \left(\frac{\mu_{2}}{\mu_{3}}\right)\right]} ; \\
C_{2}=\frac{\ln \left(\frac{\mu_{1}}{\mu_{2}}\right) \cdot\left(T_{1}+C_{3}\right) \cdot\left(T_{2}+C_{3}\right)}{\left(T_{2}-T_{1}\right)} ; \quad C_{1}=\frac{\mu_{1}}{\exp \left(C_{2} / T_{1}\right)}
\end{gathered}
$$

To account for the dependence of viscosity on the hydrodynamic pressure the Barus formula is acceptable:

$$
\mu_{p}=\mu_{0} e^{\alpha \cdot p}
$$

where $\mu_{0}$ - viscosity of the lubricant at atmospheric pressure; $p$ - hydrodynamic pressure in the lubricating film; $\alpha$ - piezoelectric coefficient of viscosity, which depends on temperature and chemical composition of lubricants.

On the base of a combination of models (28), (34) and (36) the authors propose to use a combined dependence of viscosity versus shear rate, pressure and temperature:

$$
\mu^{*}=k \cdot \dot{\gamma}^{n-1} \cdot e^{\alpha(T) \cdot p} \cdot C_{1} \cdot e^{\left(C_{2} /\left(T+C_{3}\right)\right)} .
$$

The effect of hydrodynamic pressure in the film of lubricant on the HMCh of the connecting rod bearing is reflected in the Table 4 and Fig. 11.

\begin{tabular}{|c|c|c|c|c|c|c|}
\hline $\begin{array}{c}\text { Hydromechanical } \\
\text { characteristics }\end{array}$ & $N^{*}$, & $\begin{array}{c}T, \\
{ }^{\circ} \mathrm{C}\end{array}$ & $\begin{array}{c}Q_{B}^{*}, \\
l / s\end{array}$ & $\begin{array}{c}h_{\text {min }}^{*}{ }^{\prime} \\
\mu m\end{array}$ & $\begin{array}{c}\sup p_{\max }{ }^{\prime} \\
M P a\end{array}$ & $\begin{array}{c}\inf h_{\min }, \\
\mu m\end{array}$ \\
\hline \multirow{2}{*}{ numerical value } & $610,5^{1)}$ & 105,9 & 0,02345 & 4,416 & 280,3 & 1,930 \\
& $670,42)$ & 106,9 & 0,02420 & 5,712 & 588,6 & 2,560 \\
\hline
\end{tabular}

1) - oil viscosity is independent of pressure, 2) - viscosity depends on pressure.

Table 4 . The results of the calculation of hydro-mechanical characteristics of the connecting rod bearing for the dependence of viscosity on pressure

As seen from Table 4 and Figure 11, in the case of taking into account the effect of hydrodynamic pressure on the viscosity of the lubricant, all the values of HMCh of the bearing increase. In particular, the mean-power losses increase by $8-9 \%$, the minimum film 
thickness by $20-25 \%$, the temperature by $1-2 \%$. It is important to note that the instantaneous maximum hydrodynamic pressure is increased by $50-52 \%$.
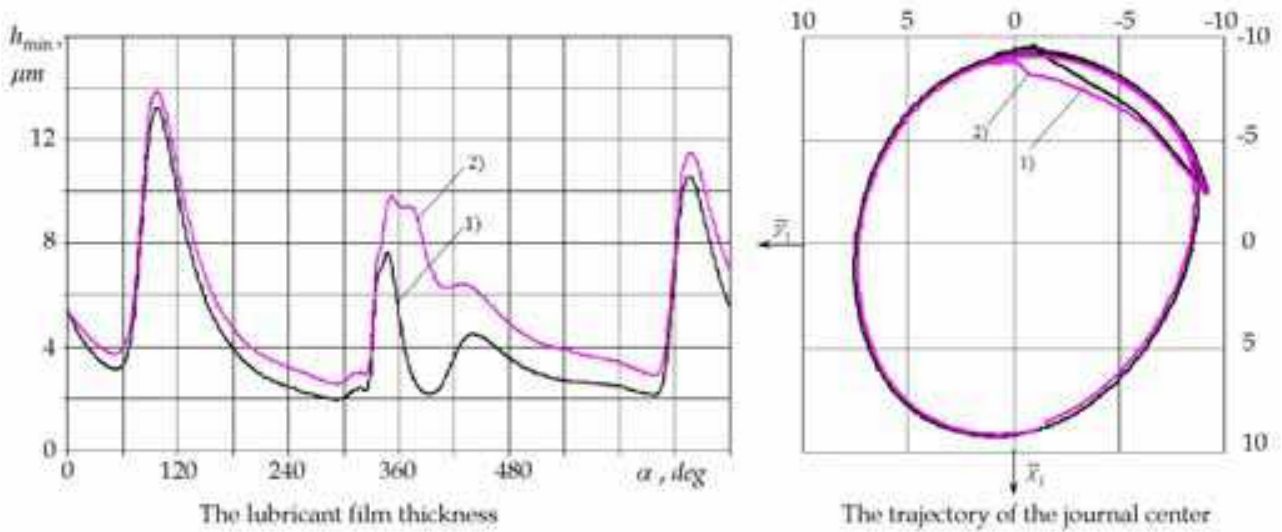

Fig. 11. The hydromechanical characteristics: 1 - Newtonian fluid, 2 - viscosity depends on pressure

Thus, accounting for one of the properties of the lubricant does not reflect the real process occurring in a thin lubricating film. Each of these properties of the lubricant and the dependence of viscosity on one of the parameters $(p, T, \dot{\gamma}, \varphi$, etc.) either improves or worsens the hydro-mechanical characteristics of tribounits. Therefore, the choice of rheological models used to calculate heavy-loaded tribounits, depends on the type of working conditions of lubricant and tribounits, as well as on the objectives pursued by the design engineer.

Further research should be focused on experimental substantiation of the parameters of rheological models, as well as the creation of calculation methods for assessing the simultaneous influence of various non-Newtonian properties of the lubricant on the dynamics of heavy loaded tribounits. This will provide simulation of real processes occurring in the lubricant film, and ultimately, will improve accuracy.

\section{Effect of elastic properties of the construction}

Elastohydrodynamic (EHD) regime of lubrication of bearings is characterized by a significant effect of dynamically changing strain of a bearing and (or) a journal on the clearance in the tribounit. Under unsteady loading the dynamic change in the geometry of the elements of tribounit caused by the finite stiffness of the bearing and the journal, leads to a change in the nature of the lubricant, hydromechanical parameters and supporting forces of tribounits and must be taken into account in the methods of its calculation.

The effect of finite stiffness of a bearing and a journal on the change of the profile of the clearance depends on the geometry of the bearing, the ratio of properties which are in contact through the lubricating film surfaces and other factors. In massive bearings local contact deformation of the surface film of the bearing and the journal prevail over the general changes of form of the bearing and the latter are usually neglected. These tribounits are usually referred to as contact-hydrodynamic (elasto-hydrodynamic). Examples of such 
units can be gears, frictionless (rolling) bearings, journal bearings with an elastic liner and rigid housing. For their calculations it is reasonable to use the methods of contact hydrodynamics (elasto-hydrodynamic lubrication theory).

However, there is also a large group of hydrodynamic friction pairs in which the general corps deformations make a significant contribution to changing the profile of the clearance. They are characterized by the presence of a continuous gradient of the deformation field, which is independent of the load location, the significant (compared with the contact and hydrodynamic tribounits) values of lubricating film thickness and values of the displacement of the friction surfaces, caused by the bending deformation of the housing, which are commensurate with them. These tribounits are called elasto-yielding (EY TU) or elastohydrodynamic. The most typical representative of the EY TU is a connecting rod bearing of a crank mechanism (crank) of engine vehicles. The desire of engine designers to maximally reduce the weight of movable elements of a crank reduces the stiffness of the bearing (crank crosshead), which makes the mode of EHD lubrication working for the connecting rod bearings. The above features - comparable with the clearance of dynamically changing elastic displacements and a continuous gradient of deformations - prevent the direct application of methods of contact-hydrodynamic lubrication theory to the calculation of EY TU.

A mathematical model of EY TU differs from the "absolutely rigid" units model by the dependence of the instantaneous value of the lubricating film thickness $h(\varphi, z, t, p)$ on the elastic displacements of the friction surface of a bearing $W(\varphi, z, t, p)$, which, in their turn, are determined by structural rigidity of the bearing and by the hydrodynamic pressure in the lubricating film $p: h(\phi, z, t, p)=h_{\text {rig }}(\phi, z, t)+W(\phi, z, t, p)$. Where $h_{\text {ri }}(\varphi, z, t)$ - film thickness in the "absolutely rigid" bearing. To determine it the expression (6) is used. Thus, the determination of pressures in the lubricating film and HMCh of EY TU is the related objective of the hydrodynamic lubrication theory and the theory of elasticity.

Modeling of EY TU, compared with "absolutely rigid" bearings is supplemented by an elastic subproblem the purpose of which is to determine the strain state of the friction surface of a crank crosshead under the influence of complex loads. The method of solving the elastic subproblem is chosen according to the accepted approximating model of an EY bearing. In today's solutions for EY TU the compliance and stiffness matrix of the bearing is usually constructed using the FE method.

The other side of modeling the elastic subsystem is adequate description of the entire complex of loads, causing the elastic deformation of the bearing housing and the conditions of fixing of FE model. One must consider not only the hydrodynamic pressure, but also the volume forces of rod inertia.

The known methods of solving the elastohydrodynamic lubrication problem can be classified as follows: direct methods or methods of successive approximations, in which the solutions of the hydrodynamic and elastic subtasks are performed separately, with the subsequent jointing of the results in the direct iterative process; and system, oriented for the joint solution of equations of fluid flow and elastic deformation.

In solving the problem of elastohydrodynamic lubrication of a bearing with the help of a direct iterative method, the hydrodynamic and elastic subproblems at each step of time discretization are solved sequentially in an iterative cycle. The main disadvantage of direct methods for the calculation of EHD is their slow convergence and the associated timeconsumption. These difficulties are partially overcome by carefully selected prediction scheme and a number of techniques that accelerate the convergence of the iterative process in the form of restrictions on movement, load and move calculation. 
Among the systemic methods the Newton-Raphson method is considered one of the most sustainable and effective solutions for elastohydrodynamic problems. In the literature it is known as the Newton-Kantorovich method or Newton (MN). The algorithm for system solutions of elastohydrodynamic problem consists of three nested iteration loops: the inner loop of implementation by the Newton method of simultaneous solution of hydrodynamic and elastic subproblems; the average - the cycle of calculation of the cavitation zone and the boundary conditions; external - the cycle of calculation of the trajectory of the journal center. Algorithm for the numerical realization of $\mathrm{MN}$ is based on the finite-difference or finite element discretization of the linearized system of equations of EHD problem (Oh\&Genka 1985; Bonneau 1995).

The application of the theory of elastohydrodynamic lubrication allows to predict lower mean-value as of the minimum lubricating film thickness as of the maximum hydrodynamic pressure. Thus, for the rod bearing of an engine, these changes may reach $35 \ldots 40 \%$. The values of the maximum hydrodynamic pressure generated in the lubricating film of a EY bearing, are also smaller than for the "absolutely rigid" one. Reduction of the maximum hydrodynamic pressure is accompanied by an increase in the size of the bearing area. This fact, together with some increase in the clearance caused by the elastic deformation of the bearing, increases the flow of lubricating fluid through the ends of the bearing. Although the pressure gradient, on which the end consumption directly depends, is reduced. The difference in the instantaneous values of the mechanical flow between "absolutely rigid" and EY TU reaches $30 \%$.

Calculation of the bearing, taking into account the elastohydrodynamic lubrication regime, not only improves the quality of design of friction units, but also clarifies the dynamic loading of mating parts such as the engine crank.

Thermoelastichydrodynamic (TEHD) regime of lubrication of journal bearings - is the mode of journal bearings, which are characterized by the influence on the magnitude of the clearance in tribounit thermoelastic deformations of a bearing and a journal, commensurate with the contribution of the displacement of the force nature.

Accounting for changes in the shape of thermoelastic friction surfaces of the journal and the bearing is possible in the case of inclusion in the resolution system of equations for the EY TU of energy equations and the relations of elasticity theory with the effects of temperature to determine the temperature fields and thermoelastic displacements caused by them. The sources of thermal fields can be either external to the tribounit, for example, a combustion chamber of an internal combustion engine for a connecting rod bearing, and internal lubricating film, in which heat generating is essential for the calculation of TEHD lubrication regime. Thus, the most complete version to solve the problem of TEHD lubrication of tribounits requires the joint consideration of problems of heat distribution in the journal, the bearing and lubricating film. The task is complicated by the fact that journal and the bearing are some idealized concepts. In reality they are rather complex shape parts (crankshaft, connecting rod, crankcase, etc.). Therefore, the methods for solving problems of TEHD lubricants are usually based on the method of FE, allowing to solve problems for bodies of complex geometric shapes the easiest.

Transient thermal fields are typical for a lubricating film of heavy-loaded bearings, which requires the simultaneous solution of equations of fluid dynamics, energy, and elasticity at each step of the calculation of trajectories. In this case, to solve all the subproblems the method of FE and schemes, similar to the systemic methods of solving the elastohydrodynamic lubrication problems, are used. 
However, practically important solution of TEHD lubrication of a tribounits is obtained for the steady thermal state, which is justified by the high inertia of the thermal fields in comparison with the rapidly changing power impacts. For calculations of tribounits with TEHD lubrication regime, this approach allows the use of a simple iterative scheme to implement solutions using the method of FE once on the preliminary stage of calculating the EY TU with regard to thermal deformations.

Nonautonomous journal bearings include bearings of multisupporting shafts of piston and rotary engines. Their distinctive feature consists in the interconnectedness of the processes occurring in various tribounits. A typical representative of nonautonomous heavy-loaded bearings is the root supports of the crankshaft of ICE. Main bearings of ICE are a part of a complex tribomechanical system, which also typically includes a crankshaft and a crankcase. Crankshaft journals are interconnected through a resilient connection - crankshaft ICE. Bushings of main bearings are installed in the holes of crankcase walls, and thus, main bearings are interconnected via a flexible design of the crankcase. Therefore, in the most general formulation to calculate the bearings it is necessary to solve a related EHD (or TEHD) problem for a system of "crankshaft - lubricating films - the crankcase". An additional feature of this system is the dependence of the loads acting on the indigenous support on the elastic properties of the crankshaft and crankcase.

In the first methods of calculation of non-autonomous main bearings of ICE a crankshaft model was used as the core spatial frame mounted on a linear-elastic mounts. The use of approximate methods for calculating the trajectories of main crankshaft journal bearings allowed to evaluate the influence of nonlinear properties of the lubricant film on the dynamics and the loading of the crankshaft. Simultaneously, we took into account the effect of necks (journals) deviation, as well as linear and bending stiffness of tribounits on the HMCh of main bearings (Zakharov, 1996a). However, to obtain the results significant estimates and approximate models of lubricating films of main bearings are used.

Currently, for the calculation of bearings the development of computer technology allows to use the exact solution of the Reynolds (4) and Elrod (1) equation for the bearing of finite length, to carry out the assessments of the impact of the crankcase and crankshaft construction supports, and of misalignments of bearings and shaft journals on HMCh. To solve such problems it is advisable to use an iterative algorithm that requires consistent calculation of loads acting on each of the bearings of the shaft and the calculation of the trajectories of its journals in the bearings.

Such calculations of the system of engine bearings allow to determine not only the optimal geometric parameters and position of sources for supplying lubricant to the main bearings, but also limiting in terms of supports performance tolerances concerning the position and shape of the friction surfaces of bearings and of the crankshaft journals.

The application of modern methods of calculation of main bearings allows to specify the values of loads acting on the crankcase and crankshaft, and their strength characteristics. But the task of elastohydrodynamic lubrication for a system "crankshaft - lubricating films the crankcase" in the most general setting is still not solved.

\section{Performance criteria}

The development of criteria for evaluating the performance of heavy-loaded hydrodynamic bearings of piston and rotary engines is an integral part of modern methods of calculation and design. At the same time, the reliability of methods themselves is largely dependent on the applied criteria (Zakharov, 1996b). Reliability of criteria is usually assessed on the base 
of comparison of calculated results with those obtained experimentally or during operation. On the basis of experimental studies and modern methods of calculation the criteria of performance of hydrodynamic tribounits are developed: the smallest allowable film thickness $h_{\text {per }}$, maximum allowable hydrodynamic pressure $p_{\text {per }}$, minimum film thickness reduced to the diameter of the journal, the maximum unit load $f_{\max }$. According to calculations of crankshaft engine bearings of several dimensions the maximum permissible loading parameters listed in the table 5 are obtained.

Assessment of performance of bearings is also done according to the calculated value of the relative total lengths of areas per the cycle of loading $\alpha_{h_{p e r}}$ and $\alpha_{p_{p e r}}$, where the values of $\inf h_{\text {min }}$ are less, and $\sup p_{\max }$ are bigger than acceptable values. Experience has shown that these parameters should not exceed $20 \%$ (Fig. 12).

\begin{tabular}{|c|c|c|c|c|c|c|c|c|c|c|c|c|}
\hline \multirow{6}{*}{ Engine group } & \multicolumn{12}{|c|}{ Loading parameters } \\
\hline & \multicolumn{4}{|c|}{$\begin{array}{l}\text { Maximum } \\
\text { specific } \\
\text { ad } f_{\max }, \mathrm{MPa}\end{array}$} & \multicolumn{4}{|c|}{$\begin{array}{l}\text { Reduced to the diameter } \\
\text { of the journal minimum } \\
\text { film thickness, } \\
\mathrm{m} \mu / 100 \mathrm{~mm}\end{array}$} & \multicolumn{4}{|c|}{$\begin{array}{l}\text { The largest } \\
\text { hydrodynamic pressure } \\
\text { in the lubricating film } \\
\sup p_{\max }, \mathrm{MPa}\end{array}$} \\
\hline & \multicolumn{12}{|c|}{ Bearing Type } \\
\hline & \multicolumn{2}{|c|}{ Crank } & \multicolumn{2}{|c|}{ Main } & \multicolumn{2}{|c|}{ Crank } & \multicolumn{2}{|c|}{ Main } & \multicolumn{2}{|c|}{ Crank } & \multicolumn{2}{|c|}{ Main } \\
\hline & \multicolumn{12}{|c|}{$\begin{array}{c}\text { Antifriction material: } \\
\text { SB - stalebronzovye inserts coated with lead bronze, } \\
\text { SA - staleallyuminievye inserts coated aluminum alloy AMO 1-20 }\end{array}$} \\
\hline & SB & SA & SB & SA & SB & SA & SB & SA & SB & SA & SB & SA \\
\hline $\begin{array}{c}\text { Highly } \\
\text { accelerated }\end{array}$ & 55 & 49 & 41 & 37 & & & 1 & & 448 & 397 & 336 & 305 \\
\hline $\begin{array}{c}\text { Medium } \\
\text { accelerated }\end{array}$ & 52 & 46 & 34 & 30,5 & & & 1 , & 5 & 420 & 377 & 275 & 245 \\
\hline $\begin{array}{c}\text { Low } \\
\text { accelerated }\end{array}$ & 45 & 39,5 & 31 & 27 & & & 1 , & 9 & 367 & 326 & 255 & 225 \\
\hline
\end{tabular}

Table 5. Maximum permissible loading parameters of sliding bearings of a crankshaft of automotive internal combustion engines
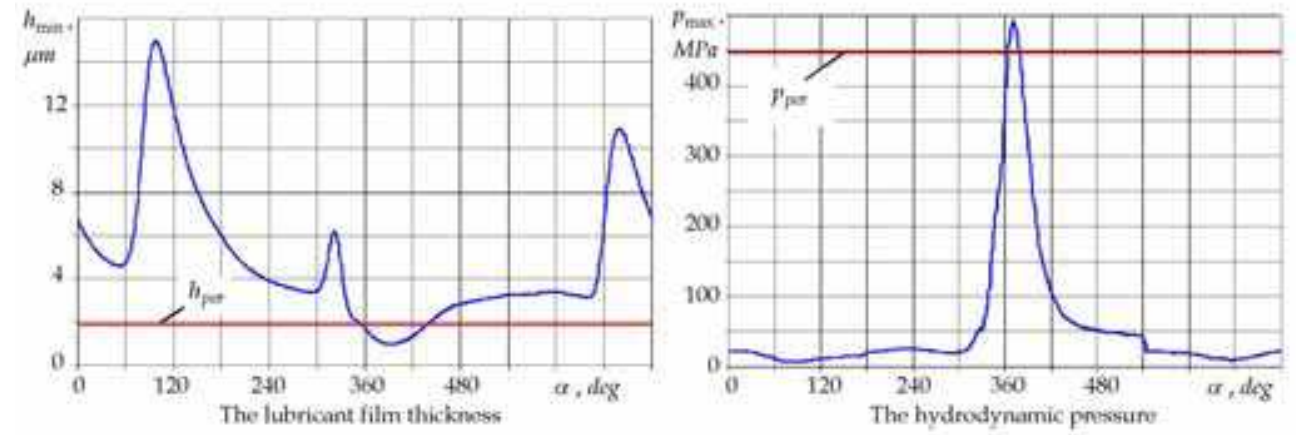

Fig. 12. The dependence of the hydromechanical characteristics on the rotation angle of crankshaft 


\section{Conclusion}

Thus, the methodology of calculating the dynamics and HMCh of heavy-loaded tribounits lubricated by structurally heterogeneous and non-Newtonian fluids, consists of three interrelated tasks: defining the field of hydrodynamic pressures in a thin lubricating film that separates the friction surfaces of a journal and a bearing with an arbitrary law of their relative motion; calculation of the trajectory of the center of the journal; the calculation of the temperature of the lubricating film.

Mathematical models used in the calculation must reflect the nature of the live load, lubricant properties, geometry and elastic properties of a construction. The choice of models is built on the working conditions of tribounits in general and the properties of the lubricant. This will allow on the early stages of the design of tribounits to evaluate their bearing capacity, thermal stress and longevity.

\section{Acknowledgment}

The presented work is executed with support of the Federal target program «Scientific and scientifically pedagogical the personnel of innovative Russia» for 2009-2013.

\section{References}

Elrod, H. (1981). A Cavitation Algorithm. Journal of lubrication Technology, Vol.103, No.3, (July 1981), pp. 354-359, ISSN 0201-8160

Prokopiev, V., Rozhdestvensky, Y. et al. (2010). The Dynamics and Lubrication of Tribounits of Piston and Rotary Machines: Ponograph the Part 1, South Ural State University, ISBN 978-5-696-04036-3, Chelyabinsk

Prokopiev, V. \& Karavayev, V. (2003). The Thermohydrodynamic Lubrication Problem of Heavy-loaded Journal Bearings by Non-Newtonian Fluids, Herald of the SUSU. A series of "Engineering", Vol.3, No 1(17), pp. 55-66

Whilkinson, U. (1964). Non-Newtonian fluids, Moscow: Mir

Gecim, B. (1990). Non-Newtonian Effect of Multigrade Oils on Journal Bearing Perfomance, Tribology Transaction, Vol. 3, No 3, pp. 384-394.

Mukhortov, I., Zadorozhnaya, E., Levanov, I. et al. (2010). Improved Model of the Rheological Properties of the Boundary layer of lubricant, Friction and lubrication of machines and mechanisms, No 5, pp. 8-19

Oh, K. \& Genka, P. (1985). The Elastohydrodynamic Solution of Journal Bearings Under Dynamic Loading, Journal of Tribology, No 3, pp. 70-76.

Bonneau, D. (1995). EHD Analysis, Including Structural Inertia Effect and Mass-Conserving Cavitation Model, Journal of Tribology, Vol. 117, (July 1995), pp. 540-547

Zakharov, S. (1996). Calculation of unsteady-loaded bearings, taking into account the deviation of the shaft and the regime of mixed lubrication, Friction and Wear, Vol.17, No 4, pp. 425-434, ISSN 0202-4977

Zakharov, S. (1996). Tribological Evaluation Criteria of Efficiency of Sliding Bearings of Crankshafts of Internal Combustion Engines, Friction and Wear, Vol.17, No 5, pp. 606 - 615, ISSN 0202-4977 


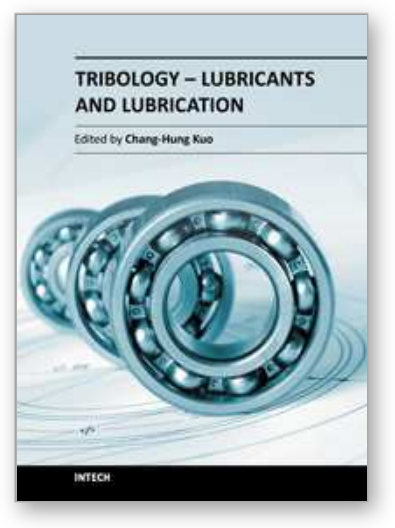

\author{
Tribology - Lubricants and Lubrication \\ Edited by Dr. Chang-Hung Kuo
}

ISBN 978-953-307-371-2

Hard cover, 320 pages

Publisher InTech

Published online 12, October, 2011

Published in print edition October, 2011

In the past decades, significant advances in tribology have been made as engineers strive to develop more reliable and high performance products. The advancements are mainly driven by the evolution of computational techniques and experimental characterization that leads to a thorough understanding of tribological process on both macro- and microscales. The purpose of this book is to present recent progress of researchers on the hydrodynamic lubrication analysis and the lubrication tests for biodegradable lubricants.

\title{
How to reference
}

In order to correctly reference this scholarly work, feel free to copy and paste the following:

Juri Rozhdestvenskiy, Elena Zadorozhnaya, Konstantin Gavrilov Igor Levanov, Igor Mukhortov and Nadezhda Khozenyuk (2011). Methodology of Calculation of Dynamics and Hydromechanical Characteristics of HeavyLoaded Tribounits, Lubricated with Structurally-Non-Uniform and Non-Newtonian Fluids, Tribology - Lubricants and Lubrication, Dr. Chang-Hung Kuo (Ed.), ISBN: 978-953-307-371-2, InTech, Available from:

http://www.intechopen.com/books/tribology-lubricants-and-lubrication/methodology-of-calculation-of-dynamicsand-hydromechanical-characteristics-of-heavy-loaded-tribounit

\section{INTECH}

open science | open minds

\section{InTech Europe}

University Campus STeP Ri

Slavka Krautzeka 83/A

51000 Rijeka, Croatia

Phone: +385 (51) 770447

Fax: +385 (51) 686166

www.intechopen.com

\section{InTech China}

Unit 405, Office Block, Hotel Equatorial Shanghai

No.65, Yan An Road (West), Shanghai, 200040, China

中国上海市延安西路65号上海国际贵都大饭店办公楼 405 单元

Phone: +86-21-62489820

Fax: $+86-21-62489821$ 
(C) 2011 The Author(s). Licensee IntechOpen. This is an open access article distributed under the terms of the Creative Commons Attribution 3.0 License, which permits unrestricted use, distribution, and reproduction in any medium, provided the original work is properly cited. 